\title{
High Resolution He-like Argon And Sulfur Spectra From The PSI ECRIT
}

\author{
M. Trassinelli*, S. Biri ${ }^{\dagger}$, S. Boucard*, D.S. Covita**, D. Gotta ${ }^{\ddagger}$, B. Leoni ${ }^{\S}$, A. Hirtl ${ }^{\mathbb{T}}$, \\ P. Indelicato*, E.-O. Le Bigot*, J.M.F. dos Santos**, L.M. Simons ${ }^{\S}$, L. Stingelin ${ }^{\S}$, \\ J.F.C.A. Veloso ${ }^{\| \dagger \dagger}$, A. Wasser ${ }^{\S}$ and J. Zmeskal ${ }^{\mathbb{I I}}$ \\ ${ }^{*}$ Laboratoire Kastler Brossel, Université Pierre et Marie Curie, Paris, France \\ ${ }^{\dagger}$ Insitute of Nuclear Research (ATOMKI), Debrecen, Hungary \\ ${ }^{*}$ Physics Department, University of Coimbra, Portugal \\ ¥Institut für Kernphysik, Forschungszentrum Jülich, Jülich, Germany \\ $\S$ Paul Scherrer Institut, Villigen PSI, Switzerland \\ IÏsterreichisch Akademie der Wissenschaften, Wien, Austria \\ $\|$ Physics Department, University of Coimbra \\ ${ }^{\dagger}$ Physics Department, University of Aveiro, Portugal
}

\begin{abstract}
We present new results on the X-ray spectroscopy of multicharged argon, sulfur and chlorine obtained with the Electron Cyclotron Resonance Ion Trap (ECRIT) in operation at the Paul Scherrer Institut (Villigen, Switzerland). We used a Johann-type Bragg spectrometer with a spherically-bent crystal, with an energy resolution of about $0.4 \mathrm{eV}$. The ECRIT itself is of a hybrid type, with a superconducting split coil magnet, special iron inserts which provides the mirror field, and a permanent magnetic hexapole. The high frequency was provided by a $6.4 \mathrm{GHz}$ microwave emitter.

We obtained high intensity X-ray spectra of multicharged F-like to He-like argon, sulfur and chlorine with one 1s hole. In particular, we observed the $1 s 2 s^{3} S_{1} \rightarrow 1 s^{2}{ }^{1} S_{0} M 1$ and $1 s 2 p^{3} P_{2} \rightarrow 1 s^{2}{ }^{1} S_{0} M 2$ transitions in He-like argon, sulfur and chlorine with unprecedented statistics and resolution. The energies of the observed lines are being determined with good accuracy using the He-like M1 line as a reference.

We surveyed the He-like M1 transition intensity as a function of the ECRIT working conditions. In particular we observed the M1 intensity dependency on the coil current and on the injected microwave power.
\end{abstract}

\section{INTRODUCTION}

The Electron Cyclotron Resonance Ion Trap (ECRIT) of the Paul Scherrer Institut (PSI) has been set up with the goal of producing narrow X-ray lines that would yield the response function of the Bragg crystal spectrometer with high accuracy [1]. Such a measurement is crucial for the ongoing pionic hydrogen experiment at PSI [2, 3, 4]. For low- to medium- $Z$ atoms, the E1 X-rays from hydrogen-like ions and the M1 X-rays from helium-like ions have an energy of a few $\mathrm{keV}$, with a natural width which is negligible compared to the expected resolution of the Bragg crystal spectrometer. The ion kinetic energy in an ECRIS is small: it is on the level of less than $1 \mathrm{eV}$ [5, 6]. Because of this, a Doppler broadening of less than $40 \mathrm{meV}$ can be expected in transitions in the $3 \mathrm{keV}$ energy range in He-like argon.

In 2002, the $1 s 2 s^{3} S_{1} \rightarrow 1 s^{2}{ }^{1} S_{0} M 1 \mathrm{X}$-ray transition in He-like argon has been used to study the response function of the Bragg crystal spectrometer with quartz
[10-1] and silicon [111] crystals [7]. In spring 2004, the characterization of the spectrometer was completed with the test of our whole set of crystals (quartz [10-1], quartz [100] and silicon [111]) using the M1 transition line from He-like argon, chlorine and sulfur. In addition, we obtained X-ray spectra of highly-charged ions of these elements with unprecedented statistics and resolution.

\section{EXPERIMENTAL SET-UP}

The experimental set-up is almost identical to the 2002 run set-up described in Ref. [7]. It is composed of the ECRIT source and of a Bragg crystal spectrometer coupled to a position sensitive detector (see fig. (1).

The ECRIT consists of a pair of a superconducting split coil magnets (which, together with special iron inserts, provides the mirror field configuration), of an AECR-U style permanent hexapole magnet, and of a $6.4 \mathrm{GHz}$ power regulated emitter. The mirror field pa- 


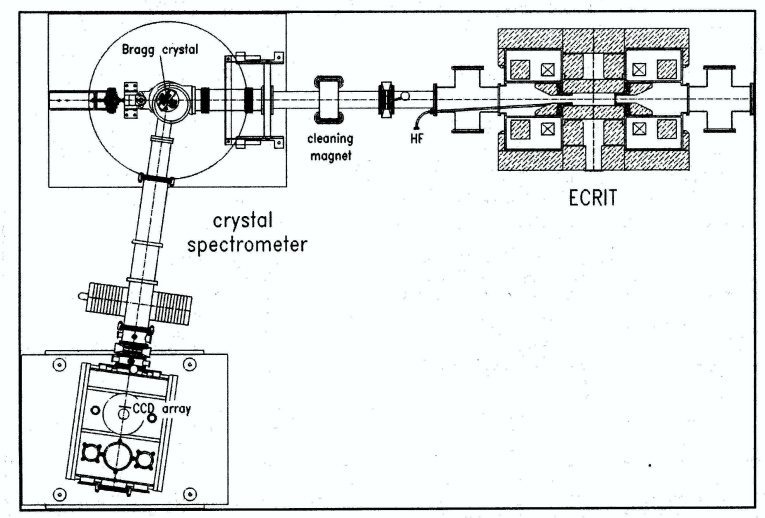

FIGURE 1. Set-up of the experiment.

rameters provide one of the highest mirror ratios for ECR sources, with a value of 4.3 over the length of the plasma chamber.

By using a cryopump and reducing the surface of iron insertion pieces the reference pressure (i.e. without plasma) was reduced from $1.7 \cdot 10^{-7} \operatorname{mbar}(2002)$ to 3 . $10^{-8}$ mbar Gas filling was supplied radially through the gaps in the open structure hexapole. The gas composition was routinely controlled and stabilized with a quadrupole mass spectrometer.

A Bragg crystal spectrometer of reflection type (Johann configuration) was installed at a distance of $2200 \mathrm{~mm}$ from the plasma center. Silicon and quartz crystal have used for the 2004 run, with different Bragg angle values. The crystals are $100 \mathrm{~mm}$ diameter circular plates, with a thickness of $0.2-0.3 \mathrm{~mm}$, and are spherically bent with a curvature radius of $2982.4 \pm 0.6 \mathrm{~mm}$, by optical attachment to high-quality quartz spherical lenses.

The detector is an array of 6 CCDs of $600 \times 600$ pixels each [8], with an energy resolution of $140 \mathrm{eV}$ at $3 \mathrm{keV}$. The pixel size at working temperature $\left(-100^{\circ} \mathrm{C}\right)$ has been recently measured to be $39.9943 \pm 0.0035 \mu \mathrm{m}$ [9]. The granularity of the detector was decisive in discriminating charged particle events against X-rays possessing different topologies. The CCD chips and the associated electronics were protected against light and highfrequency (HF) power by a $30 \mu m$ thick Beryllium window installed in the vacuum tube between the crystal spectrometer and the ECRIT.

\section{ECRIT at work}

During spring 2004, we injected different kinds of gases in the ECRIT in order to obtain X-ray spectra from highly-charged argon, chlorine and sulfur. For this propose, we used a gas mixture of $\mathrm{O}_{2}$ and, respectively, $\mathrm{Ar}, \mathrm{CHClF}_{2}$ and $\mathrm{SO}_{2}$. Using the experience acquired in the previous run, we adjusted a mixing ratio of around $1: 9$, with a total pressure in the plasma chamber of $3-$ $4 \cdot 10^{-7}$ mbar. In order to recognize the different charge states, we used as an initial reference the $K \alpha$ or $K \beta$ lines of the neutral gas, which are easily recognizable: they are the brightest when only a few watts of HF power are injected. Using known energy intervals, we were then able to move the spectrometer to the region of the nearby $1 s 2 s^{3} S_{1} \rightarrow 1 s^{2}{ }^{1} S_{0} M 1$ transition in the He-like ion, and to observe it. We then optimized the different ECRIT and spectrometer parameters in order to maximize the line intensity in the detector. The typical illumination time of the CCD chips before the readout is $1 \mathrm{~min}$. Due to the large intensity of the X-rays, this allows for a sizeable probability of double hits for pixels near the line peak. In order to reduce this effect and improve the peak-to-background ratio, a densimet ${ }^{\circledR}$ collimator was inserted at a distance of $150 \mathrm{~mm}$ from the center of the plasma, leaving an aperture of $28 \mathrm{~mm}(\mathrm{~h}) \times 4 \mathrm{~mm}(\mathrm{v})$ or $28 \mathrm{~mm}(\mathrm{~h}) \times 1 \mathrm{~mm}(\mathrm{v})$, depending on the configuration. During the different runs the (double hits) to (single hits) ratio always stayed below $5 \%$, which is small enough to properly handle double hits in the final analysis, and to neglect triple hit processes.

During the optimization of the apparatus, we studied the M1 line intensity as a function of the injected HF power. As expected, we observed a strong dependence between the M1 intensity and the HF power (see fig. 2). In contrast, we noted an unexpected behavior of the maxima of the curves, whose HF intensity does not increase with the ionization energy of the He-like ion.

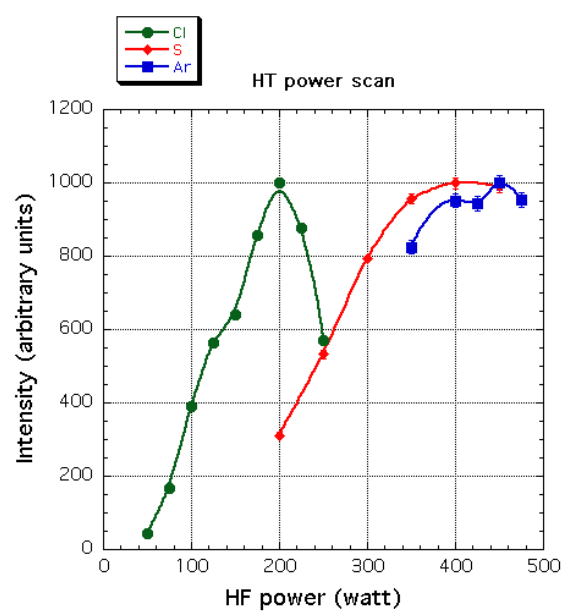

FIGURE 2. Injected HT power scan for argon, chlorine and sulfur versus the He-like M1 intensity (in arbitrary units). 


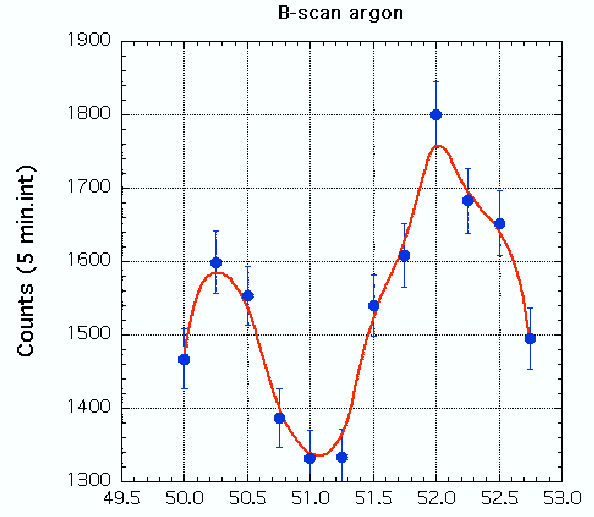

I(A)

FIGURE 3. He-like argon M1 line intensity dependency versus the superconducting coils current. $55 \mathrm{~A}$ corresponds to a magnetic field value of $2.35 \mathrm{kG}$ at the center of the ECRIT.

During the ECRIT parameters adjustment, we observed a non-trivial dependency of the M1 intensity against the longitudinal magnetic field. As shown in fig. 3. we observe the presence of two distinct maxima in the M1 intensity-coil current relationship.

\section{Atomic Spectra}

One of the most important goals of the spring 2004 run was the high-precision measurement of the X-ray spectra of argon, chlorine and sulfur. With 1-2 hours maximum acquisition time, we obtained high-statistics spectra of He-, Li- and Be-like ionic states of these elements. A crystal spectrometer like ours can only measure energy differences between atomic transitions. A reference is thus needed. Due to the lack of high quality reference line in neutral atom X-ray spectra (see, e.g., [10]) we used as a reference He-like $1 s 2 s^{3} S_{1} \rightarrow 1 s^{2}{ }^{1} S_{0} M 1$ transition. All the transition energies provided in the present work are based on the M1 theoretical transition energy calculated with a multi-configuration Dirac-Fock code [11, 12]. The peaks in the spectra were fitted with a simulated spectrometer response function which was convoluted to a Gaussian. The response function was obtained through a Monte Carlo X-ray tracking simulation based on the theoretical reflection function of the crystal obtained with the XOP code [13]. The reliability of the simulation had previously been tested during the crystal response function study [7]. The results obtained in highly-charged argon and sulfur spectroscopy have an unprecedented precision of the order of $10 \mathrm{meV}$ and they agree with the previous experimental values and theoretical predictions (see tables 12 .

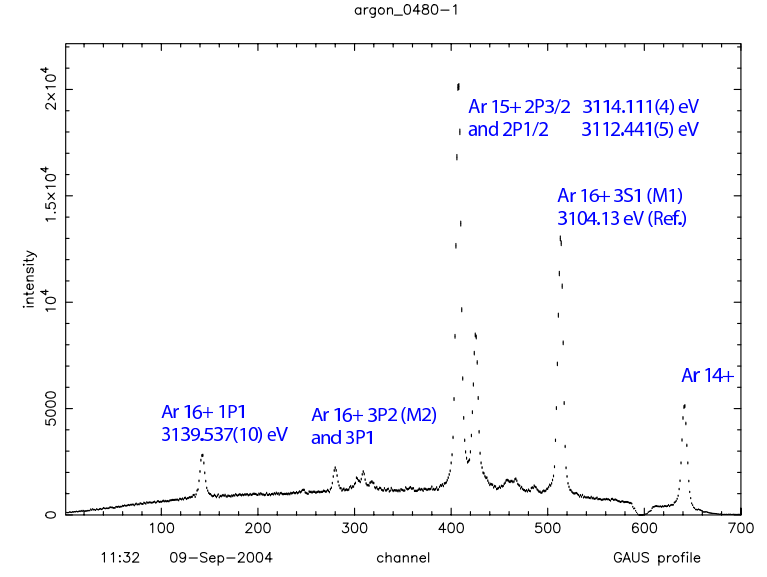

FIGURE 4. He-like and Li-like argon spectrum using quartz [10-1] crystal. HF power injected $P=400 \mathrm{~W}$. argon partial pressure $p_{A r}=5 \cdot 10^{-9} \mathrm{mbar}$, total pressure $p_{t o t}=5 \cdot 10^{-7} \mathrm{mbar}$

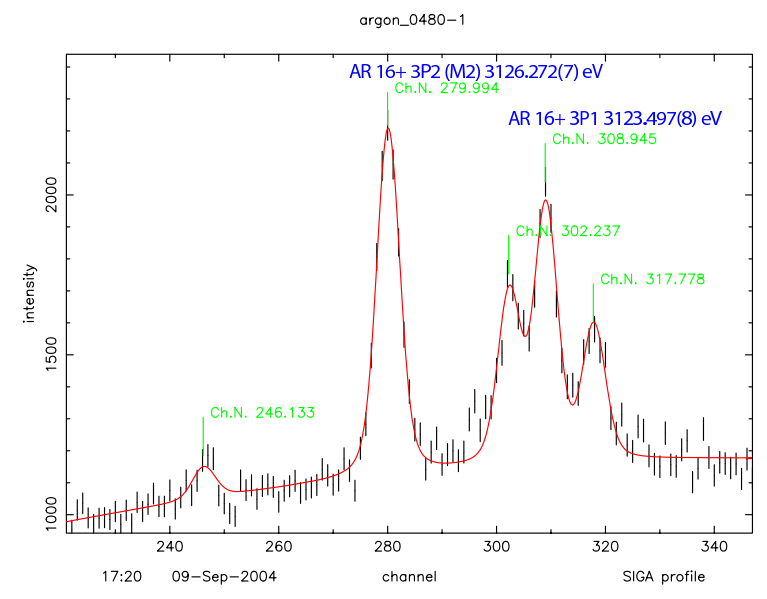

FIGURE 5. Detail of the spectrum in figure 4 around the $1 s 2 p^{3} P_{n} \rightarrow 1 s^{2}{ }^{1} S_{0}$ transitions

\section{OUTLOOK AND CONCLUSION}

In this paper, we demonstrated once more the possibility of using high-precision X-ray spectroscopy with a Bragg spectrometer in the study of ECR ion sources. Moreover, we presented new results on X-ray transition energies in highly-charged argon, chlorine and sulfur. 
TABLE 1. He-like argon energy transitions in $\mathrm{eV}$

\begin{tabular}{lll}
\hline & $\begin{array}{c}1 s 2{ }^{1} P_{1} \rightarrow \\
\rightarrow 1 s^{2}{ }^{1} S_{0}\end{array}$ & $\begin{array}{l}1 s 2 p^{1} P_{1} \rightarrow \\
\rightarrow 1 s 2 p^{3} P_{1}\end{array}$ \\
\hline Costa [11] (th.) & 3139.57 & 16.05 \\
Plante [14] (th.) & 3139.6236 & 16.0484 \\
Lindgren [15] (th.) & & 16.048 \\
Deslattes [16] (exp.) & $3139.553(36)$ & $16.031(72)$ \\
This Work & $3139.537(10)$ & $16.040(17)$ \\
(prelminary results) & & \\
\hline
\end{tabular}

TABLE 2. He-like sulfur energy transitions in $\mathrm{eV}$

\begin{tabular}{lll}
\hline & $\begin{array}{c}1 s 2 p^{1} P_{1} \rightarrow \\
\rightarrow 1 s^{2} S_{0}\end{array}$ & $\begin{array}{l}1 s 2 p^{1} P_{1} \rightarrow \\
\end{array}$ \\
& $\rightarrow 1 s 2 p^{3} P_{1}$ \\
\hline MCDF [12] (th.) & 2460.6169 & 13.4875 \\
Plante [14] (th.) & 2460.6707 & 13.4857 \\
Schleinkofer [17] (exp.) & $2460.67(9)$ & $13.62(20)$ \\
This Work & $2460.608(9)$ & $13.483(16)$ \\
(prelminary results) & & \\
\hline
\end{tabular}

The next steps will consist in finishing the data analysis of lower-charge states (Li- and Be-like) of argon and sulfur, in analyzing the chlorine spectra and in studying the injected HF power dependency of the satellite transition in the He-like ions spectra.

\section{ACKNOWLEDGMENTS}

The technical support of L. Stohwasser, H. Schneider and D. Stückler was essential in obtaining the results described here. The advice and help of D. Hitz and K. Stiebing during the preparation of the ECRIT experiment is warmly acknowledged.

\section{REFERENCES}

1. Gotta, D., Prog. Part. Nucl. Phys., 52, 133-195 (2004).

2. Biri, S., Simons, L., and Hitz, D., Rev. Sci. Instrum., 71, 1116-18 (2000).

3. Anagnostopoulos, D. F., Biri, S., Boisbourdain, V., Demeter, M., Borchert, G., Egger, J. P., Fuhrmann, H., Gotta, D., Gruber, A., Hennebach, M., Indelicato, P., Liu, Y. W., Manil, B., Markushin, V. E., Marton, H., Nelms, N., Rusi El Hassanii, A., Simons, L. M., Stingelin, L., Wasser, A., Wells, A., and Zmeskal, J., Nucl. Instrum. Methods B, 205, 9-14 (2003).

4. Pionic Hydrogen Collaboration, PSI experiment proposal $R$-98.01 (1998), URL http://pihydrogen.web.psi.ch

5. Bernard, C., Ph.D. thesis, Université J. Fourier, Lyon (1996).

6. Sadeghi, N., Nakano, T., Trevor, D. J., and Gottscho, R. A., J. Appl. Phys., 70, 2552 (1991).
7. Anagnostopoulos, D., Biri, S., Fuhrmann, H., Gotta, D., Gruber, A., Indelicato, P., Leoni, B., Simons, L. M., Stingelin, L., Wasser, A., and Zmeskal, J., eprint: physics/0408081 (2004).

8. Nelms, N., Anagnostopoulos, D. F., Ayranov, O., Borchert, G., Egger, J. P., Gotta, D., Hennebach, M., Indelicato, P., Leoni, B., Liu, Y. W., Manil, B., Simons, L. M., and Wells, A., Nucl. Instrum. Meth. A, 484, 419-31 (2002).

9. Trassinelli, M., "Precision Spectroscopy Of Pionic Atoms: From Pion Mass Evauation To Tests Of Chiral Perturbation Theory," in DAФNE 2004 proceeding, Frascati Physics Series, to be published, eprint: physics/0409066.

10. Anagnostopoulos, D. F., Gotta, D., Indelicato, P., and Simons, L. M., Phys. Rev. Lett., 91, 240801 (2003).

11. Costa, A. M., Martins, M. C., Parente, F., Santos, J. P., and Indelicato, P., At. Data Nucl. Data Tables, 79, 223-39 (2001).

12. Indelicato, P., private communication (2004).

13. Sanchez del Rio, M., and Dejus, J., "XOP: Recent development," in SPIE proceedings, 1998, p. 3448.

14. Plante, D., Johnson, W., and Sapirstein, J., Phys. Rev. A, 49, 3519-3530 (1994)

15. Lindgren, I., Åsén, B., Salomonson, S., and MårtenssonPendrill, A. M., Phys. Rev. A, 64, 062505 (5) (2001).

16. Deslattes, R., Beyer, H., and Folkmann, F., J. Phys. B: At. Mol. Opt. Phys., 17, L689-L694 (1984).

17. Schleinkofer, L., Bell, F., Betz, H., Trolman, G., and Rothermel, J., Phys. Scr., 25, 917-923 (1982). 